PROCEEDINGS OF THE

AMERICAN MATHEMATICAL SOCIETY

Volume 131, Number 9, Pages 2933-2936

S 0002-9939(03)07136-3

Article electronically published on April 9, 2003

\title{
MEASURES INVARIANT UNDER THE GEODESIC FLOW AND THEIR PROJECTIONS
}

\author{
CRAIG J. SUTTON
}

(Communicated by Michael Handel)

\begin{abstract}
Let $S^{n}$ be the $n$-sphere of constant positive curvature. For $n \geq 2$, we will show that a measure on the unit tangent bundle of $S^{2 n}$, which is even and invariant under the geodesic flow, is not uniquely determined by its projection to $S^{2 n}$.
\end{abstract}

\section{INTRODUCTION}

The topological entropy, $h_{T}(g)$, of a Riemannian manifold $(M, g)$ is a geometric invariant which attempts to capture the complexity of the geodesic flow. In KKW91 it was shown that for a metric $g$ of negative sectional curvature the function $h_{T}\left(g_{\lambda}\right)$ is $C^{1}$, where $g_{\lambda},-\epsilon<\lambda<\epsilon$, is a $C^{2}$-perturbation of $g$, and an explicit formula for the derivative was obtained. As an application of this formula they established the following interesting result.

Theorem 1.1 ([KKW91, pp. 21 and 28]). Let $M$ be a compact surface and let $\mathcal{R}(M)$ denote the submanifold of negatively curved $C^{2}$ metrics on $M$ having area equal to 1 . Then $h_{T}: \mathcal{R}(M) \rightarrow \mathbb{R}$ has a critical point at $g_{0}$ if and only if the Lebesgue measure $l_{g_{0}}$ and the Margulis measure $\mu_{g_{0}}$ with respect to $g_{0}$ have the same projection to $M$; that is, $l_{g_{0}}$ and $\mu_{g_{0}}$ agree on $\pi^{-1}(\mathcal{B}(M))=\left\{\pi^{-1}(A): A \in \mathcal{B}(M)\right\}$, where $\mathcal{B}(M)$ is the $\sigma$-algebra of Borel subsets on $M$ and $\pi: S(M) \rightarrow M$ is the canonical projection.

Katok, Knieper and Weiss went on to conjecture that for an arbitrary compact manifold $(M, g)$ of negative sectional curvature the Margulis and Lebesgue measures with respect to $g$ coincide whenever they have the same projection to $M$. As they note, establishing this conjecture would then demonstrate Theorem 1.1 in arbitrary dimensions [KKW91, p. 21].

The above results led Flaminio to consider the general problem of determining the measures on the unit tangent bundle which are invariant under the geodesic flow and are determined by their projection to $M$. By restricting his attention to the class of even measures, that is, measures on $S(M)$ which are invariant under the flip map $(x, v) \stackrel{\sigma}{\rightarrow}(x,-v)$ on $S(M)$, Flaminio obtained the following.

Received by the editors October 1, 2001.

2000 Mathematics Subject Classification. Primary 53D25.

Key words and phrases. Geodesic flows, differential geometry.

(C)2003 American Mathematical Society 
Theorem 1.2 ([Fla92]). Let $g$ be a metric of positive sectional curvature on $S^{2}$. Then an even, $G_{t}$-invariant distribution $T$ is determined by its projection to $S^{2}$. That is, $T$ is determined by its values on the set

$$
\pi^{*}\left(C^{\infty}\left(S^{2}\right)\right) \equiv\left\{f \circ \pi: f \in C^{\infty}\left(S^{2}\right)\right\},
$$

where $\pi: S\left(S^{2}\right) \rightarrow S^{2}$ is the natural projection.

In particular, this result shows that for a closed surface $(M, g)$ of positive sectional curvature the even, $G_{t}$-invariant probability measures are determined by their projections to $M$. It is natural to wonder whether this result generalizes to all closed Riemannian manifolds of positive sectional curvature. By studying the right regular representation of $S O(n)$ we obtain the following negative answer.

Theorem 1.3. Let $\left(S^{2 j}, g\right)$ be the standard sphere of constant curvature 1 with $j \geq 2$. Then even, $G_{t}$-invariant complex measures on $S\left(S^{2 j}\right)$ are not determined by their projection to $S^{2 j}$. In particular, there are non-zero, even, $G_{t}$-invariant finite real measures on $S\left(S^{2 j}\right)$ which project to zero on $S^{2 j}$.

\section{Constructing the measure}

By the Riesz representation theorem there is an isomorphism between bounded linear functionals and complex measures. For a linear functional $F: L^{2}(S(M)) \rightarrow \mathbb{C}$ the notion of projecting to $M$ translates into restricting $F$ to the set $\pi^{*}\left(L^{2}(M)\right) \equiv$ $\left\{f \circ \pi: f \in L^{2}(M)\right\}$. The notions of evenness and $G_{t}$-invariance are also defined in the obvious way for $F$. Consequently, we see that we can construct measures as in Theorem 1.3 by finding a linear functional with the corresponding properties. This will be carried out in the remainder of this paper.

For our discussion we fix the following notation.

A) $G=\mathrm{SO}(n)$.

B) $H=\mathrm{SO}(2) \oplus I_{n-2}$.

C) $K=I_{2} \oplus \mathrm{SO}(n-2)$.

D) $L=[1] \oplus \mathrm{SO}(n-1)$.

E) $L^{2}(G, d x)=\left\{f: G \rightarrow \mathbb{C}\right.$ measureable : $\left.\int_{G}\|f\|^{2} d x<\infty\right\}$; where $d x$ is Haar measure.

F) $G$ will act on $L^{2}(G, d x)$ via the right regular representation $\Phi: G \rightarrow$ $\operatorname{Aut}\left(L^{2}(G, d x)\right)$, which is given by $(\Phi(g) . f)(x)=f(x g)$.

G) For any representation $(V, \tau)$ of an arbitrary group $B$ we let $V^{S}=\{v \in V$ : $\tau(s) . v=v$ for all $s \in S\}$ for any $S \subset B$.

We also note that for the sphere $S^{n-1}=G / L$ of constant positive sectional curvature 1 the geodesic flow is given by the right action of $H$ on $S\left(S^{n-1}\right)=G / K$ and the flip map $\sigma: S\left(S^{n-1}\right) \rightarrow S\left(S^{n-1}\right)$ can be realized as the right action of [1] $\oplus-I_{n-1}$ on $G / K$ when $n$ is odd.

In constructing the desired measure we will find the following lemma to be useful.

Lemma 2.1. Let $n=2 j+1 \geq 5$ and let $S$ be the subgroup of $G$ generated by $H, K \leq G$ and $\sigma \in G$. Then there exists a finite dimensional unitary representation $\tau: G \rightarrow \mathrm{GL}(W)$ such that $\left(W^{L}\right)^{\perp} \cap W^{S} \neq\{0\}$. In particular, we may take $(W, \tau)$ to be an irreducible representation of $G$.

Indeed, let $(W, \tau)$ be an irreducible representation of $G$ as in Lemma 2.1. Then $W$ can be thought of as a subrepresentation of $L^{2}(G)$. Using this identification and 
taking $\theta \in\left(W^{L}\right)^{\perp} \cap W^{S}$ we may define $\tilde{F}: L^{2}(G) \rightarrow \mathbb{C}$ by

$$
f \mapsto \int_{G} f \bar{\theta} d x
$$

It then follows from the Peter-Weyl theorem (see [Kna86]) and the way $\theta$ was chosen that $\tilde{F}$ has the following properties.

(1) $\tilde{F} \mid L^{2}(G)^{L} \equiv 0$.

(2) $\tilde{F} \mid L^{2}(G)^{S} \not \equiv 0$. In particular, $\tilde{F} \mid L^{2}(G)^{K} \not \equiv 0$.

Now, since $L^{2}(G)^{K}$ can be indentified with $L^{2}(G / K)$ we see from property (2) that $\tilde{F}$ actually defines a non-zero bounded linear functional $F: L^{2}(G / K) \rightarrow \mathbb{C}$, which is even and invariant under the geodesic flow. Furthermore, it follows from property (11) that the projection of $F$ to $S^{n-1}=G / L$ is zero. Then as noted earlier the Riesz representation theorem provides us with a non-zero, even, $G_{t}$-invariant complex measure on $S\left(S^{n-1}\right)$ that projects to zero. Consequently, one of the real measures $\operatorname{Re}(\mu)$ or $\operatorname{Im}(\mu)$ also has these properties. Now, all that remains to be done is to prove Lemma 2.1

Proof of Lemma 2.1. We let $\mathfrak{g}, \mathfrak{l}, \mathfrak{h}, \mathfrak{k}$ and $\mathfrak{s}$ denote the Lie algebras of $G, L, H$, $K$, and $S$ respectively. Following an argument due to G. Prasad we will show that Ad $: G \rightarrow \operatorname{Aut}\left(\operatorname{Sym}^{2}\left(\mathfrak{g}^{\mathbb{C}}\right)\right)$ is a representation of $G$ which satisfies Lemma 2.1, where $\mathfrak{g}^{\mathbb{C}}=\mathfrak{g} \oplus i \mathfrak{g}$ is the complexification of $\mathfrak{g}$ and Ad is the natural linear extension of the adjoint representation of $G$.

Upon inspection we can see that $\mathfrak{s}=\mathfrak{h} \oplus \mathfrak{k}$ and $\mathfrak{g}=\mathfrak{l} \oplus \mathbb{R}^{n-1}=\mathfrak{s} \oplus \mathbb{R}^{2 n-2}$. From this we can see that

$$
\mathfrak{g}^{\mathbb{C}}=\mathfrak{l}^{\mathbb{C}} \oplus\left(\mathbb{R}^{n-1}\right)^{\mathbb{C}}=\mathfrak{h}^{\mathbb{C}} \oplus \mathfrak{k}^{\mathbb{C}} \oplus\left(\mathbb{R}^{2 n-2}\right)^{\mathbb{C}} .
$$

Hence,

$$
\begin{aligned}
\operatorname{Sym}^{2}\left(\mathfrak{g}^{\mathbb{C}}\right) & =\operatorname{Sym}^{2}\left(\mathfrak{l}^{\mathbb{C}}\right) \oplus \operatorname{Sym}^{2}\left(\left(\mathbb{R}^{n-1}\right)^{\mathbb{C}}\right) \oplus\left(\mathfrak{l}^{\mathbb{C}} \otimes\left(\mathbb{R}^{n-1}\right)^{\mathbb{C}}\right) \\
& =\operatorname{Sym}^{2}\left(\mathfrak{l}^{\mathbb{C}}\right) \oplus \operatorname{Sym}^{2}\left(\left(\mathbb{R}^{n-1}\right)^{\mathbb{C}}\right) \oplus \operatorname{Hom}\left(\mathfrak{l}^{\mathbb{C}},\left(\mathbb{R}^{n-1}\right)^{\mathbb{C}}\right)
\end{aligned}
$$

and

$$
\operatorname{Sym}^{2}\left(\mathfrak{g}^{\mathbb{C}}\right)^{L}=\operatorname{Sym}^{2}\left(\mathfrak{l}^{\mathbb{C}}\right)^{L} \oplus \operatorname{Sym}^{2}\left(\left(\mathbb{R}^{n-1}\right)^{\mathbb{C}}\right)^{L} \oplus \operatorname{Hom}\left(\mathbb{l}^{\mathbb{C}},\left(\mathbb{R}^{n-1}\right)^{\mathbb{C}}\right)^{L} .
$$

Since $\left(\operatorname{Ad}_{L}, \mathbb{l}^{\mathbb{C}}\right)$ and $\left(\operatorname{Ad}_{L},\left(\mathbb{R}^{n-1}\right)^{\mathbb{C}}\right)$ are inequivalent irreducible representations of $L$ we see that $\operatorname{Hom}\left(\mathfrak{l}^{\mathbb{C}},\left(\mathbb{R}^{n-1}\right)^{\mathbb{C}}\right)^{L}=0$. Otherwise we would have a non-zero, $\mathbb{C}$ linear map $T: \mathfrak{l}^{\mathbb{C}} \rightarrow\left(\mathbb{R}^{n-1}\right)^{\mathbb{C}}$ such that $\operatorname{Ad}_{L}(x) \circ T=T \circ \operatorname{Ad}_{L}(x)$ for all $x \in L$. It would then follow from Schur's lemma that $T$ would have to be an isomorphism, which would contradict the non-equivalence of the representations. Therefore,

$$
\operatorname{Sym}^{2}\left(\mathfrak{g}^{\mathbb{C}}\right)^{L}=\operatorname{Sym}^{2}\left(\mathfrak{l}^{\mathbb{C}}\right)^{L} \oplus \operatorname{Sym}^{2}\left(\left(\mathbb{R}^{n-1}\right)^{\mathbb{C}}\right)^{L} .
$$

We now recall the following well-known fact.

Lemma 2.2. Let $G$ be a compact toplogical group and $(\tau, V)$ a finite dimensional irreducible $\mathbb{C}$-representation. Then $\operatorname{dim}_{\mathbb{C}} \operatorname{Sym}^{2}(V)^{G}=1$. That is, up to a scalar multiple there is a unique Hermitian inner product $\omega \in \operatorname{Sym}^{2}(V)$ on $V$ with respect to which $(\tau, V)$ is unitary.

Hence, it follows that $\operatorname{dim}_{\mathbb{C}} \operatorname{Sym}^{2}\left(\mathfrak{g}^{\mathbb{C}}\right)^{L}=2$. Also, since

$$
\begin{aligned}
\operatorname{Sym}^{2}\left(\mathfrak{g}^{\mathbb{C}}\right)^{S}= & \operatorname{Sym}^{2}\left(\mathfrak{h}^{\mathbb{C}}\right)^{S} \oplus \operatorname{Sym}^{2}\left(\mathfrak{k}^{\mathbb{C}}\right)^{S} \oplus \operatorname{Sym}^{2}\left(\left(\mathbb{R}^{2 n-2}\right)^{\mathbb{C}}\right)^{S} \oplus \operatorname{Hom}\left(\mathfrak{h}^{\mathbb{C}}, \mathfrak{k}^{\mathbb{C}}\right)^{S} \\
& \oplus \operatorname{Hom}\left(\mathfrak{h}^{\mathbb{C}},\left(\mathbb{R}^{2 n-2}\right)^{\mathbb{C}}\right)^{S} \oplus \operatorname{Hom}\left(\mathfrak{k}^{\mathbb{C}},\left(\mathbb{R}^{2 n-2}\right)^{\mathbb{C}}\right)^{S}
\end{aligned}
$$


a similar argument shows that $\operatorname{dim}_{\mathbb{C}} \operatorname{Sym}^{2}\left(\mathfrak{g}^{\mathbb{C}}\right)^{S}=3$. Putting all of this together we see that $\operatorname{dim}_{\mathbb{C}}\left(\operatorname{Sym}^{2}\left(\mathfrak{g}^{\mathbb{C}}\right)^{L}\right)^{\perp}=\operatorname{dim}_{\mathbb{C}} \operatorname{Sym}^{2}\left(\mathfrak{g}^{\mathbb{C}}\right)-2$ and $\operatorname{dim}_{\mathbb{C}} \operatorname{Sym}^{2}\left(\mathfrak{g}^{\mathbb{C}}\right)^{S}=3$, which implies

$$
1 \leq \operatorname{dim}_{\mathbb{C}}\left(\left(\operatorname{Sym}^{2}\left(\mathfrak{g}^{\mathbb{C}}\right)^{L}\right)^{\perp} \cap \operatorname{Sym}^{2}\left(\mathfrak{g}^{\mathbb{C}}\right)^{S}\right) \leq 3,
$$

which proves our lemma.

We point out that our dimension argument fails when $n=3$ - as it should by Theorem [1.2. In this case $H=\left\langle I_{3}\right\rangle$ and $L=[1] \oplus S O(2)$. Hence, $\mathfrak{s}=\mathfrak{k} \oplus \mathfrak{h}=$ $\mathfrak{k} \cong \mathfrak{s o}(2) \cong \mathfrak{l}$, which implies that $\operatorname{dim}_{\mathbb{C}} \operatorname{Sym}^{2}\left(\mathfrak{g}^{\mathbb{C}}\right)^{S}=2=\operatorname{dim}_{\mathbb{C}} \operatorname{Sym}^{2}\left(\mathfrak{g}^{\mathbb{C}}\right)^{L}$. This prevents the last line of our argument from working.

\section{REFERENCES}

[Fla92] Livio Flaminio. Une remarque sur les distribution invariantes par les flots géodésiques des surface. C.R. Acad. Sci. Paris, Série I, 315:735-738, 1992. MR 94a:58156

[KKW91] A. Katok, G. Knieper, and H. Weiss. Formulas for the derivative and critical points of topological entropy for Anosov and geodesic flows. Comm. Math. Phys., 138:19-31, 1991. MR 92d:58162

[Kna86] Anthony W. Knapp. Representation Theory of Semisimple Lie Groups: An Overview Based on Examples. Princeton University Press, 1986. MR 87j:22022

Department of Mathematics, University of Michigan, Ann Arbor, Michigan 48103

Current address: Department of Mathematics, University of Pennsylvania, 209 South 33rd Street, Philadelphia, Pennsylvania 19104-6395

E-mail address: cjsutton@math.upenn.edu 